\title{
THE LANGUAGES OF PARASITE COMMUNICATION
}

\author{
Isabel Roditi*
}

*Institute of Cell Biology, University of Bern, Baltzerstrasse 4,

$\mathrm{CH}-3012$,

Bern, Switzerland

Email: isabel.roditi@izb.unibe.ch 


\section{ABSTRACT}

Although it is regarded as self-evident that parasites interact with their hosts, with the primary aim of enhancing their own survival and transmission, the extent to which unicellular parasites communicate with each has been severely underestimated. Recent publications show that information is commonly exchanged between parasites of the same species and that this can govern their decisions to divide, to differentiate or to migrate as a group. Communication can take the form of soluble secreted factors, extracellular vesicles or contact between cells. Extracellular parasites can do this directly, while intracellular parasites use the infected host cell - or components derived from it - as an intermediary. By emitting signals that can be dispersed within the host, parasites can also have long-distance effects on the course of an infection and its pathology. This article presents an overview of recent developments in this field and draws attention to some older work that merits re-examination. 
Most readers of this article will not need to be told that diseases such as sleeping sickness, malaria, Leishmaniasis, Trichomoniasis and Chagas Disease are caused by unicellular parasites. This knowledge might come with the tacit assumption that singlecelled organisms are completely self-sufficient and have no need to interact, much less cooperate, with members of their own species. In recent years, however, it has become apparent that a number of decisions are group decisions that rely on parasites communicating with each other. Just as animals can exchange information in different ways, be it vocally, visually, chemically or by body language, parasites have also developed a range of mechanisms for communicating with each other. This sometimes occurs directly, from parasite to parasite, or by using the infected host cell - or components derived from it - as an intermediary. By emitting signals that can be dispersed within the host, parasites can also have wide-ranging effects on the course of an infection and its pathology. This article presents an overview of recent developments in this field and draws attention to some older work that merits re-examination. Owing to space constraints, it will not cover molecules used by intracellular parasites to reprogram the cells that they infect.

\section{Quorum sensing and differentiation}

Different subspecies of Trypanosoma brucei, the pathogens responsible for human sleeping sickness and Nagana in domestic animals, are extracellular throughout their life cycle. In the mammalian host the parasites use a quorum sensing mechanism to maintain a balance between slender bloodstream forms, which are capable of dividing every 6-8 hours, and stumpy bloodstream forms, which cannot divide and have a lifespan of a few days. When trypanosomes ingested by a tsetse fly, the slender form is killed while the stumpy form survives and differentiates to the next life stage, the procyclic form, in the insect midgut $[1,2]$. If slender forms were to proliferate unchecked, this would result in rapid death of the mammalian host. If all were to 
differentiate to stumpy forms, however, there would only be a short window when transmission to the fly could take place. It is thus in the parasite's interest to promote a chronic infection in which both life-cycle stages occur, and this is best achieved through population-wide communication. Slender forms release one or more molecules $<500 \mathrm{Da}$ collectively known as stumpy induction factor (SIF) [3]. When the titre of these cells is high, the concentration of SIF exceeds a critical threshold, triggering differentiation of slender to stumpy forms. Once cells become stumpy, they no longer produce SIF and its concentration wanes. These cycles of SIF production and cell differentiation, superimposed on the phenomenon of antigenic variation, result in the characteristic waves of parasitaemia in an infected host. In cultures treated with SIF, the number of cells expressing stumpy markers can approach one hundred per cent [3], but this does not happen in vivo otherwise the infection would extinguish itself. It could be that a sub-population of slender forms (termed slender retainers by Mony and Matthews [4]) is refractory to SIF, or that local concentrations of SIF differ. Several regulators of SIF have been identified: the kinases MAPK5, ZFK and TOR4 [5-7] all make trypanosomes less receptive to the factor, while the RNA-binding protein RBP7, a MAP kinase kinase and several other proteins participate in stumpy formation and the progression to procyclic forms $[4,8]$. Thus, subtle differences in the balance between the pathways promoting maintenance as slender forms and progression to stumpy forms might determine the fate of a cell. SIF can be mimicked by cell-permeable analogues of cAMP that are subsequently hydrolysed to AMP [9]. However, despite being functionally characterised close to two decades ago, the identity of SIF is still not known nor has the receptor been identified.

In contrast to T. brucei, the replication of Plasmodium falciparum is intracellular. Sporozoites introduced with the saliva of an infected mosquito invade hepatocytes and undergo a first round of differentiation and replication, giving rise to tens of thousands of merozoites per infected cell. When released into the bloodstream, these can undergo multiple cycles of invasion and replication in red blood cells and cause the characteristic symptoms of malaria. In some red blood cells, however, there is a switch from 
merozoite production to gametocytogenesis. As a result, male and female gametocytes are released into the circulation, from where they can be taken up by mosquitoes during a blood meal. Once again, there needs to be a balance between virulent replicative forms and transmissible forms, and once again there seems to be a quorum sensing mechanism that can tip the equilibrium towards gametocyte production. In this case, however, the messengers are membrane-bound vesicles that are released by infected red blood cells; these are termed red blood cell microvesicles [10] or exosome-like vesicles [11] by the two groups that discovered them. Although both groups report the same effect on gametocyte production, the vesicles they characterised differed in size. It is not known whether this is due to differences in experimental protocols for cell culture or in the protocols for their isolation. Release of these vesicles peaks in late schizogony $[10,11]$ and is independent of parasite egress [10]. Both host and parasite proteins are present in the vesicles and their composition, together with the requirement for the parasite protein PfPTP2, is consistent with them being derived from Maurer's clefts and specific sub-domains of the red blood cell membrane $[10,11]$. Once released, the vesicles can bind to both infected and uninfected erythrocytes, but internalisation appears to be specific to infected cells [10]. Again, it seems that not all cells are equally receptive, since fewer than half the infected cells take up microvesicles, even when they are present at very high concentrations [10]. As is the case with SIF, the active components of the vesicles have yet to be identified. It is conceivable that several components act in concert and these might be proteins, RNA and/or metabolites. It is also possible that additional signals contribute to commitment to gametocytogenesis.

In connection with the phenomenon of vesicle transfer, Regev-Rudzki et al. reported that vesicles could transmit DNA between infected erythrocytes and confer drug resistance to other parasites [11]. In some instances the transferred DNA was episomal, but in one case it was integrated into the parasite genome and was presumed to have looped out. One way for gene transfer to occur would be if the contents of the nucleus were sampled during vesicle formation and delivered to the parasite nucleus in the recipient cell. To date, however, there have been no reports that the vesicles 
contain nucleoproteins $[10,12]$. Alternatively, DNA would have to leave the nucleus and traffic to the Maurer's clefts in order to be incorporated into vesicles. It is still an open question if gene transfer of this type occurs in nature and to what effect.

\section{Coordinated group movement - the phenomenon of social motility}

When stumpy forms of T. b. brucei are taken up by the tsetse fly, they differentiate into procyclic forms in the endoperitrophic space [13]. Early procyclic forms can be recognised by their expression of a GPI-anchored protein, GPEET procyclin, which is expressed exclusively by this stage of the life cycle $[14,15]$. Within a week of ingestion, the parasites differentiate to late procyclic forms that are GPEET-negative [14, 15]. In addition to this surface marker, a number of other proteins are differentially expressed between the two forms [16]. The differentiation from early to late procyclic forms coincides with the movement of the parasites to the ectoperitrophic space, but it is not known whether the parasites first migrate, then differentiate, or vice versa. Early procyclic forms show a striking change in behaviour, known as social motility (SoMo) when they are transferred from liquid culture onto plates containing agarose [17]. When a small volume of a culture is spotted onto a plate, the cells initially remain in a confined region at the inoculation site. As well as dividing, which they do approximately once a day [16], they form communities that can move across the surface and recruit other individuals [17]. When the population rises above 1.5 million cells, the communities start to form radial projections that elongate at a rate of $10 \mathrm{~mm}$ a day (equivalent to 500 body lengths) $[16,17]$. Individual cells within the projections are not constrained, but appear to move freely in all directions [17]. As long as they do not encounter a barrier, the projections continue to extend and gradually spiral clockwise [17]. The directionality of group movement is most likely due to the structure of the parasite's flagellum, which possesses an inherent chirality [18]. For wild-type communities, the radial projections are usually symmetrically distributed around the periphery of the central colony [16]. This indicates that early procyclic forms produce and respond to a migration factor, which may also determine the spacing between projections. The fact that the cells need 
to reach a certain number before migration starts is again indicative of quorum sensing and a response mechanism that requires a threshold concentration.

Two proteins are required for the production or activity of the migration factor. These are phosphodiesterase B1 (PDEB1), which is localised along the length of the flagellum [19] and Rft1, an endoplasmic reticulum protein that plays a role in $\mathrm{N}$-linked glycosylation [20]. Chemical inhibition of phosphodiesterases or depletion of PDEB1 by RNAi completely abolishes SoMo [19]. Knocking out Rft1 causes a less severe defect the cells need to reach a higher threshold number before they start migrating and they produce fewer projections [20]. Mixing PDEB1 knockdown mutants with wild-type cells overcomes the defect, indicating that the downstream signalling cascade is still intact [19]. Negative regulators of SoMo have also been identified: knocking down the procyclic form-specific adenylate cyclases ACP1/2 and ACP6 result in a hypersocial phenotype, with the production of more radial projections than in communities expressing wild-type levels [21]. Interestingly, ACP1 and ACP6 both localise to the flagellar tip [22]. Taken together with the phenotype of the PDEB1 knockdown, these data indicate that increases in local concentrations of cAMP inhibit SoMo. Other adenylate cyclases are differentially regulated between early and late procyclic forms, but it is not known if they play a role in SoMo [16]. All trypanosomal adenylate cyclases have a large extracellular domain, a single trans-membrane domain and an intracellular catalytic domain [23]. No ligands that regulate their activities have been identified. Might the extracellular domains provide a means by which trypanosomes interact directly with each other and, by causing changes in adenylate cyclase activity, transmit signals between cells? This could potentially allow them to coordinate their movements.

Late procyclic forms do not exhibit social motility [16]. Moreover, when a culture consists of a mixture of early and late procyclic forms, the late forms accumulate at the inoculation site [16]. This indicates that they do not react to migration factors; it is not known whether or not they still produce them. In addition to the factors that coordinate migration and group movement, both early and late procyclic forms release repellents [19]. These are active over considerable distances (equivalent to hundreds of body 
lengths) and they cause the leading edges of radial projections to reorient and avoid the source. However, two migrating streams can move in parallel lines that are very close to each other $[19,24]$. This indicates that the sides and the tip of the projections differ in their perception of signals. One way of visualising this is to think of each projection (as

well as the flagellum of every trypanosome that contributes to it) as a form of elephant's trunk; in this case it is obvious that the tip that has a very different structure and function to the sides. At present, there are no known mutants with altered production or perception of the repellents, and we know little about these factors except that they can be transferred between plates and are non-volatile (S. Imhof, S. Millius and IR, unpublished). Given the distances that they cover, one possibility is that they are very small molecules that can diffuse rapidly through the agarose matrix. Another possibility is that they might be released in vesicles that move over the surface. Such vesicles might interact with recipient cells or they might break down after some time and release their contents. In this way they would not repel the population that produced them.

As intriguing as it is, how pertinent is SoMo to trypanosome biology? If it reflects part of the life cycle in the fly, one would predict that mutants defective in migration on plates would also show a defect in establishing infections. This is the case for the Rft1 knockout, which is the only mutant to have been tested in vivo so far [20]. While one mutant may not be sufficient confirm a hypothesis beyond all doubt, it has certainly made us think differently. We are all inclined to view transmission as an interaction between the trypanosome and its host; it is now time to consider whether communication between parasites is also part of the equation.

\section{Can communication between parasites drive cell division?}

As we have seen above, when SIF reaches a critical threshold, bloodstream forms differentiate and undergo cell-cycle arrest. But can cells also signal to each other to divide? Procyclic forms of Trypanosoma brucei are the life-cycle stages that develop and proliferate in the tsetse midgut. These are normally grown in liquid culture and can reach densities exceeding $10^{7}$ per $\mathrm{ml}$ [25]. While it might not seem obvious that 
trypanosomes within a culture are communicating with each other, there are several indications that they do so. First of all, some strains of trypanosomes are sensitive to culture density. Procyclic forms of T. b. brucei AnTat 1.1, for example, exhibit a lag in growth if they are diluted below $10^{6}$ cells $\mathrm{ml}^{-1}$ [25]. Second, when procyclic forms are cloned they require conditioned medium from a logarithmically growing culture. This suffices for some strains, but other strains also requires companion cells that gradually die out as the transformed cells grow up during selection [25]. What is being provided by conditioned medium and companion cells? The glib explanation, that they provide "growth factors", needs some critical appraisal. What are the cells releasing and transmitting to each other? From the way in which conditioned medium is prepared vesicles/exosomes could be present, as well as freely soluble components. It is also conceivable that trypanosomes are communicating by direct cell-cell contact and that this contributes to the decision to divide or not.

Another example of cell-cell interactions stimulating replication is provided by Theileria parva, the parasites causing East Coast fever in cattle [26]. Like Plasmodium, T. parva is an apicomplexan parasite that replicates intracellularly. When a sporozoite invades a lymphocyte it develops into a multinucleated schizont in the host cytoplasm. In culture, T. parva-infected (Tpi) cells can proliferate indefinitely as long as the parasite is present. Treatment with the theilericidal drug BW720C causes the lymphocytes to revert to a quiescent phenotype [27]. Tpi cells require at least two signals for optimal growth. These are provided by conditioned medium and by surface components on other cells [28]. Interleukin 2 is one of the growth-stimulating components in conditioned medium, but there may be others, including microvesicles or exosomes. Fixed cells or membrane preparations can substitute for living cells, indicating that the signals they provide are not simply due to secretion of cytokines [28]. While the stimulatory lymphocytes do not need to be infected with Theileria, and could thus be bystander cells in the host, the ability to respond by proliferation requires the presence of the parasite [28]. In cattle, Tpi cells form microfoci in both lymphoid and nonlymphoid tissues; in this way they could mutually deliver and receive stimuli. Why 
should parasites want company in order to replicate? One explanation might be that cell division is costly, and the presence of other parasites indicates that the environment is worth this investment.

\section{Parasite-derived vesicles for long-range communication with the host}

It has been known for decades that parasites interact with their hosts and modulate the immune response. This was thought to be due to direct interactions between cell surfaces or to the production of secreted/excreted molecules that could reach host cells and alter their function. The definition of excreted molecules was rather vague, essentially encompassing all molecules that did not appear to follow classical secretion pathways. In the past few years, however, several parasites have been shown to produce membrane-bound vesicles that increase their chances of survival and transmission [10-12, 29-34]. In theory, there are many ways in which the contents of these vesicles can be delivered to cells within the host. They can fuse with host cell membranes, be taken up by endocytosis or they can act like controlled-delivery capsules that discharge their contents at different times and in different locations that might be far away from the parasite itself. The packaging of contents in vesicles may prevent their recognition and neutralisation by the host's immune system. Alternatively, when parasite proteins are delivered to uninfected cells they might stimulate an immune response; depending on the context, this might be beneficial or detrimental to the host.

Trichomonas vaginalis is a sexually transmitted parasite that grows within the human urogenital tract. The parasite is extracellular and colonisation entails attachment to epithelial cells. In addition to these direct interactions between the parasite and the host, T. vaginalis sheds exosomes ranging in size from 50 - $150 \mathrm{~nm}$ [29]. These contain many orthologues of proteins in mammalian exosomes, as well as parasite-specific proteins and small RNAs. T. vaginalis exosomes can fuse with host epithelial cells and release their cargo. This alters the production of two pro-inflammatory cytokines, with an increase in interleukin 6 and a decrease in interleukin 8. It is possible that this constellation enables the parasite to maintain a chronic infection. Three parasite surface 
molecules have been implicated in attachment: a lipoglycan that binds to galectins on the host cell surface [35] and two membrane proteins (TVAG_244130 and TVAG_166850) that are abundant in highly adherent strains [36]. Ectopic expression of either protein in a less adherent strain results in a two-fold increase in attachment to ectocervical epithelial cells. Intriguingly, exosomes derived from a highly adherent strain enhance attachment of a poorly adherent strain. Neither TVAG_244130 nor TVAG_166850 were identified in the exosome proteome [29], indicating that other molecules must effect the increase. Why should an efficiently adhering parasite support the attachment of a less efficient strain? Is it altruism on the part of the former, opportunism on the part of the latter, or mutual aid? A clue may come from the genderspecific differences observed by Twu and coworkers, with some strains enhancing attachment to vaginal epithelial cells and others to prostate epithelial cells [29]. Given that transmission of $T$. vaginalis is frequently heterosexual, this strategy might enable the parasite to optimise colonisation in both male and female hosts.

Another example of parasite-secreted vesicles increasing adhesion and infection is provided by Trypanosoma cruzi, the agent of Chagas' disease [37]. This parasite releases membrane-bound vesicles by at least two different mechanisms [38]. Larger vesicles (ectosomes) bud from the plasma membrane, while smaller vesicles (exosomes) are found in the flagellar pocket. Vesicles purified from epimastigotes, the proliferative forms in the insect vector, and metacyclics, which are the infective forms for mammals, showed differences in both their protein and small RNA contents, suggesting that they have specialised functions [38,39]. Pretreatment of mice with parasite-derived vesicles can alter the outcome of infection, resulting in an increased amastigote burden and an intensified inflammatory response in the heart (the latter being manifested by increased levels of IL4 and IL10 mRNA and infiltration by macrophages and CD4 ${ }^{+}$lymphocytes). [40]. Since the vesicles were administered intraperitoneally in these experiments, this suggests that they were either capable of homing in to the correct tissue or that they elicited a cascade of events that increased the susceptibility of heart cells to infection. Exposure to extracellular vesicles can modulate the transcriptome of mammalian cells 
[41]. As gauged by microarray analysis, incubation of HeLa cells with extracellular vesicles altered the expression levels of several hundred mRNAs. This was most apparent 6-24 hours post exposure, with most transcripts returning to basal levels after $72 \mathrm{~h}$ [41]. The RNA contents of the extracellular vesicles shed by T. cruzi are mainly derived from rRNAs and a restricted subset of tRNA fragments (tsRNAs) [42].

Furthermore, parasites transfected with Cy3-labelled tsRNAs are able to transfer these to other parasites and to mammalian cells [42]. Reprogramming of host cell expression cannot be attributed to these alone, however; transfection of HeLa cells with individual tsRNAs resulted in many fewer changes in transcript levels and, for the most part, these were not concordant with the results obtained with vesicles [41].

As mentioned above, extracellular vesicles from Plasmodium-infected red blood cells can stimulate gametocyte formation. In addition, they can modulate the innate immune response. Vesicles from erythrocytes infected with $P$. falciparum or $P$. berghei have been shown to stimulate the production of IL6 and TNF by peripheral blood monocytes and macrophages, respectively $[10,30]$. Some parameters were tested in only one of the two systems and it is not yet clear if they will hold for other species of Plasmodium. For example, vesicles from infections with $P$. falciparum also stimulated IL $\beta$, IL12 and the anti-inflammatory cytokine IL10. That the cytokine response required uptake of vesicles was demonstrated for $P$. falciparum, but for no other species to date. Conversely, the requirement for TLR4 and MyD88 was established only for $P$. berghei [30].

If extracellular vesicles are transferred to an uninfected host, can they alter the course of a subsequent infection? An attenuated strain of $P$. yoelii preferentially infects reticulocytes, whereas a virulent strain infects mature red blood cells. Experiments with exosomes derived from the attenuated strain indicate that they can have a protective effect against the virulent strain [12]. This was most marked when an adjuvant, CpG oligodeoxynucleotide, was administered in conjunction with the exosomes, protecting most mice against lethal challenge. The effect was not simply one of preventing infection, but changed the target host cell from red blood cells to reticulocytes. Most 
mice were able to clear this infection, however, and subsequently remained free of parasites.

Parasites of the genus Leishmania, which cause a wide spectrum of diseases, replicate extracellularly in their sandfly hosts, and intracellularly in their mammalian hosts. Infection of mammals is initiated by metacyclic promastigotes, which are regurgitated in a plug of promastigote secretory gel (PSG) when the sand fly takes a blood meal [43, 44]. The parasites first pass through neutrophils [45] before infecting macrophages and differentiating to amastigotes. Replication occurs within a parasitopherous vacuole and culminates in the release of amastigotes that can also invade macrophages and undergo further cycles of replication. Both insect- and parasite-derived components enhance the replication of transmitted parasites $[34,46-$ 48]. Two biological activities have been attributed to filamentous proteophosphoglycan, a major component of PSG. One is the ability to recruit macrophages to the inoculation site; the other is to stimulate the conversion of arginine to polyamines, which are required for growth of Leishmania, rather than nitric oxide, which activates macrophages and promotes parasite killing [49]. Leishmania promastigotes were among the first examples of parasites that were shown to extrude exosomes in culture [31-33]. In common with extracellular vesicles from Trichomonas and T. cruzi, these are enriched for specific fragments of tRNAs and rRNAs [33]. With one exception, similar cargoes were identified in exosomes from $L$. donovani and $L$. brasiliensis; the latter is competent for RNA interference and also packages small interfering RNAs [33]. Very recently, it was demonstrated that exosomes with the same composition as culture-derived exosomes are produced by parasites in the sand fly and that these enhance the parasite load when co-injected with parasites [34]. In common with the other organisms described above, exosomes from Leishmania manipulate cytokine secretion, in this case enhancing the production of pro-inflammatory cytokines IL8 and IL17a, while inhibiting the production of TNF $\alpha$, IL10 and IL12p70 [31, 32]. It has also been reported that infection with $L$. donovani lowers the expression of a microRNA, miR-122, in the liver, resulting in lowered serum cholesterol [50]. The reduction in 
steady-state miR-122 was attributed to the delivery of the surface protease gp63 to liver cells by exosomes, resulting in the cleavage of Dicer1 [50]. No other microRNAs were analysed, so is not known if this is a specific or a general phenomenon.

Extracellular vesicles have also been implicated in the pathology of African trypanosomes. The possible release of microvesicles via the flagellar pocket of $T$. brucei was first reported by Geiger and coworkers during their characterisation of the parasite secretome [51]. Very recently, it was shown that bloodstream forms of T. brucei extrude nanotubes from the flagellar pocket and that these convert to extracellular vesicles that have the capacity to fuse with both parasite and erythrocyte membranes [52]. The vesicles are enriched for flagellar proteins, but also contain proteins derived from other cell compartments. Extracellular vesicles derived from parasites that are resistant to human serum (through expression of SRA) increased the survival of serumsensitive parasites in vitro, consistent with delivery of functional SRA to the endosomal compartment of the recipient. While this is an elegant proof of protein transfer between parasites, it is an open question whether sufficient SRA would be transferred during a natural infection where trypanosomes are present at much lower titres and the concentrations of lytic factors are higher than are seen in a laboratory setting. Severe anaemia is a feature of bovine trypanosomiasis $[53,54]$. It is most obvious in infections caused by T. congolense, but is also observed when T. brucei reaches high titres. More than a quarter of a century ago it was observed that anaemia in the early phase of infection could be reversed by treatment with trypanocidal drugs, indicating that it relied on the continuous presence of parasites or parasite-derived factors [53]. Szempruch and coworkers demonstrated that extracellular vesicles altered the membrane properties of erythrocytes and, when injected into mice, resulted in a $20 \%$ decrease in erythrocytes within an hour [52]. No further decrease was detected at 24 hours, however, which might mean that the lytic effect of purified vesicles was local and/or transient. Another explanation might be that not all red blood cells in the circulation are equally vulnerable. In cattle, the genetic makeup of the host certainly 
plays a role as well, since trypanotolerant animals control anaemia better than susceptible breeds $[54,55]$.

In summary, the production of membrane-bound vesicles, which may have different sub-cellular origins, is a common feature of unicellular parasites. These are frequently, although not invariably, used to reprogram the host's innate immune system to the parasite's own benefit. Their cargoes can consist of hundreds of protein and RNA species and, for the most part, it is not known which components are responsible for their manifold effects.

\section{Direct communication between parasites}

All of the phenomena described so far entail the release of components into the wider milieu, without any certainty that they will reach their intended targets. To date, there are only two examples of structures that make direct contacts between parasites. Following activation of Plasmodium gametocytes, the parasites form closed, nanotubelike filaments that attach to the surface of other cells [56]. These filaments, which are derived from the plasma membrane, contain actin and are covered with gamete-specific proteins. Filament formation was observed in the mosquito midgut as well as in culture, indicating that it is a natural process, most probably to facilitate contact between gametes and subsequent fertilisation [56]. An unusual form of direct communication has recently been described for procyclic form trypanosomes [57]. This involves flagellar membrane fusion and the rapid exchange of proteins between connected cells, without any transfer of DNA [57]. The one prerequisite for exchange is that the proteins gain access to the flagellum. To date, the actual fusion event has only been observed in culture, but there is circumstantial evidence that it might also occur in the tsetse salivary glands. When flies were co-infected with trypanosomes expressing cytoplasmic red fluorescent protein or a tagged form of the meiotic marker HOP1, double-positive parasites were observed, compatible with protein exchange occurring before the formation and fusion of gametes [58]. Might this form of cell-cell communication occur in other flagellated parasites? Co-infection of sandflies with two tagged forms of 
Leishmania can give rise to double-positive parasites $[59,60]$. It is not always possible to isolate genetic hybrids, however [59], and one possibility is that some of them are products of protein exchange rather than mating. It is also worth noting that the Leishmania amastigote - so named because this life-cycle stage was supposed to lack a flagellum - has a flagellar pocket and a very short flagellum with a 9+0 axoneme structure that is more reminiscent of the sensory cilium of higher eukaryotes [61]. The amastigote resides within a parasitophorous vacuole in the host macrophage. It is intriguing that the tip of its flagellum can be in close contact with the vacuolar membrane [61] and one can speculate that if this provides a means of exporting proteins to the host cell.

\section{Multilingual parasites?}

As described above, parasites can communicate via small molecules, secreted proteins and proteoglycans, membrane-bound vesicles and cell-cell contact (Figure 1). Some parasites are already known to employ more than one language, and it is only a matter of time before more parasites are shown to do so. In addition, many protocols that are employed empirically to optimise growth, such as the use of conditioned medium or provision of companion cells, may simply be a way of allowing parasites to sense if relatives are in their vicinity and to react accordingly. Taking a lesson from bacteria, which are also proficient linguists [62], it would not be surprising if parasites employ many other means of communication that still await discovery. Intercellular bridges for the transfer of proteins and nutrients $[63,64]$, or ion channels that propagate waves of electrical signalling between cells [65] are just two possibilities. And given the propensity of parasites to diverge from the mainstream, it would not be surprising if they have developed entirely novel means of encrypting and exchanging information.

\section{Acknowledgments}

Gaby Schumann, Arunasalam Naguleswaran and Sebastian Millius are thanked for reading the manuscript and for stimulating discussions. Work in our laboratory is 
funded by the Swiss National Science Foundation and the Howard Hughes Medical Institute.

\section{REFERENCES}

1. Robertson M. Notes on the Polymorphism of Trypanosoma gambiense in the Blood and Its Relation to the Exogenous Cycle in Glossina palpalis. Proc Roy Soc B. 1912:527-5.

2. McLintock LM, Turner CM, Vickerman K. Comparison of the effects of immune killing mechanisms on Trypanosoma brucei parasites of slender and stumpy morphology. Parasite Immunol. 1993;15(8):475-80. PubMed PMID: 8233562.

3. Vassella E, Reuner B, Yutzy B, Boshart M. Differentiation of African trypanosomes is controlled by a density sensing mechanism which signals cell cycle arrest via the cAMP pathway. J Cell Sci. 1997;110 ( Pt 21):2661-71. PubMed PMID: 9427384.

4. Mony BM, Matthews KR. Assembling the components of the quorum sensing pathway in African trypanosomes. Mol Microbiol. 2015;96(2):220-32. doi:

10.1111/mmi.12949. PubMed PMID: 25630552; PubMed Central PMCID: PMCPMC4403954.

5. Vassella E, Kramer R, Turner CM, Wankell M, Modes C, van den Bogaard M, et al. Deletion of a novel protein kinase with PX and FYVE-related domains increases the rate of differentiation of Trypanosoma brucei. Mol Microbiol. 2001;41(1):33-46. PubMed PMID: 11454198.

6. Domenicali Pfister D, Burkard G, Morand S, Renggli CK, Roditi I, Vassella E. A Mitogen-activated protein kinase controls differentiation of bloodstream forms of Trypanosoma brucei. Eukaryot Cell. 2006;5(7):1126-35. doi: 10.1128/EC.00094-06. PubMed PMID: 16835456; PubMed Central PMCID: PMCPMC1489280.

7. Barquilla A, Saldivia M, Diaz R, Bart JM, Vidal I, Calvo E, et al. Third target of rapamycin complex negatively regulates development of quiescence in Trypanosoma brucei. Proc Natl Acad Sci U S A. 2012;109(36):14399-404. doi:

10.1073/pnas.1210465109. PubMed PMID: 22908264; PubMed Central PMCID: PMCPMC3437835.

8. Mony BM, MacGregor P, Ivens A, Rojas F, Cowton A, Young J, et al. Genome-wide dissection of the quorum sensing signalling pathway in Trypanosoma brucei. Nature. 2014;505(7485):681-5. doi: 10.1038/nature12864. PubMed PMID: 24336212; PubMed Central PMCID: PMCPMC3908871.

9. Laxman S, Riechers A, Sadilek M, Schwede F, Beavo JA. Hydrolysis products of cAMP analogs cause transformation of Trypanosoma brucei from slender to stumpy-like forms. Proc Natl Acad Sci U S A. 2006;103(50):19194-9. doi: 10.1073/pnas.0608971103. PubMed PMID: 17142316; PubMed Central PMCID: PMCPMC1748198.

10. Mantel PY, Hoang AN, Goldowitz I, Potashnikova D, Hamza B, Vorobjev I, et al. Malaria-infected erythrocyte-derived microvesicles mediate cellular communication within the parasite population and with the host immune system. Cell Host Microbe. 
2013;13(5):521-34. doi: 10.1016/j.chom.2013.04.009. PubMed PMID: 23684304; PubMed Central PMCID: PMCPMC3687518.

11. Regev-Rudzki N, Wilson DW, Carvalho TG, Sisquella X, Coleman BM, Rug M, et al. Cell-cell communication between malaria-infected red blood cells via exosome-like vesicles. Cell. 2013;153(5):1120-33. doi: 10.1016/j.cell.2013.04.029. PubMed PMID: 23683579.

12. Martin-Jaular L, Nakayasu ES, Ferrer M, Almeida IC, Del Portillo HA. Exosomes from Plasmodium yoelii-infected reticulocytes protect mice from lethal infections. PLoS One. 2011;6(10):e26588. doi: 10.1371/journal.pone.0026588. PubMed PMID: 22046311 ; PubMed Central PMCID: PMCPMC3202549.

13. Vickerman K, Tetley L, Hendry KA, Turner CM. Biology of African trypanosomes in the tsetse fly. Biol Cell. 1988;64(2):109-19. PubMed PMID: 3067793.

14. Vassella E, Den Abbeele JV, Butikofer P, Renggli CK, Furger A, Brun R, et al. A major surface glycoprotein of Trypanosoma brucei is expressed transiently during development and can be regulated post-transcriptionally by glycerol or hypoxia. Genes Dev. 2000;14(5):615-26. PubMed PMID: 10716949; PubMed Central PMCID: PMCPMC316419.

15. Acosta-Serrano A, Vassella E, Liniger M, Kunz Renggli C, Brun R, Roditi I, et al. The surface coat of procyclic Trypanosoma brucei: programmed expression and proteolytic cleavage of procyclin in the tsetse fly. Proc Natl Acad Sci U S A. 2001;98(4):1513-8. doi: 10.1073/pnas.041611698. PubMed PMID: 11171982; PubMed Central PMCID: PMCPMC29288.

16. Imhof S, Knusel S, Gunasekera K, Vu XL, Roditi I. Social motility of African trypanosomes is a property of a distinct life-cycle stage that occurs early in tsetse fly transmission. PLoS Pathog. 2014;10(10):e1004493. doi: 10.1371/journal.ppat.1004493. PubMed PMID: 25357194; PubMed Central PMCID: PMCPMC4214818.

17. Oberholzer M, Lopez MA, McLelland BT, Hill KL. Social motility in african trypanosomes. PLoS Pathog. 2010;6(1):e1000739. doi: 10.1371/journal.ppat.1000739. PubMed PMID: 20126443; PubMed Central PMCID: PMCPMC2813273.

18. Rodriguez JA, Lopez MA, Thayer MC, Zhao Y, Oberholzer M, Chang DD, et al. Propulsion of African trypanosomes is driven by bihelical waves with alternating chirality separated by kinks. Proc Natl Acad Sci U S A. 2009;106(46):19322-7. doi: 10.1073/pnas.0907001106. PubMed PMID: 19880745; PubMed Central PMCID: PMCPMC2780783.

19. Oberholzer M, Saada EA, Hill KL. Cyclic AMP Regulates Social Behavior in African Trypanosomes. MBio. 2015;6(3):e01954-14. doi: 10.1128/mBio.01954-14. PubMed PMID: 25922395; PubMed Central PMCID: PMCPMC4436052.

20. Imhof S, Vu XL, Butikofer P, Roditi I. A Glycosylation Mutant of Trypanosoma brucei Links Social Motility Defects In Vitro to Impaired Colonization of Tsetse Flies In Vivo. Eukaryot Cell. 2015;14(6):588-92. doi: 10.1128/EC.00023-15. PubMed PMID: 25862152; PubMed Central PMCID: PMCPMC4452570.

21. Lopez MA, Saada EA, Hill KL. Insect stage-specific adenylate cyclases regulate social motility in African trypanosomes. Eukaryot Cell. 2015;14(1):104-12. doi: 
10.1128/EC.00217-14. PubMed PMID: 25416239; PubMed Central PMCID: PMCPMC4279026.

22. Saada EA, Kabututu ZP, Lopez M, Shimogawa MM, Langousis G, Oberholzer M, et al. Insect stage-specific receptor adenylate cyclases are localized to distinct subdomains of the Trypanosoma brucei flagellar membrane. Eukaryot Cell. 2014;13(8):1064-76. doi: 10.1128/EC.00019-14. PubMed PMID: 24879126; PubMed Central PMCID: PMCPMC4135804.

23. Naula C, Schaub R, Leech V, Melville S, Seebeck T. Spontaneous dimerization and leucine-zipper induced activation of the recombinant catalytic domain of a new adenylyl cyclase of Trypanosoma brucei, GRESAG4.4B. Mol Biochem Parasitol. 2001;112(1):19-28. PubMed PMID: 11166383.

24. Imhof S, Roditi I. The Social Life of African Trypanosomes. Trends Parasitol. 2015;31(10):490-8. doi: 10.1016/j.pt.2015.06.012. PubMed PMID: 26433252.

25. McCulloch R, Vassella E, Burton P, Boshart M, Barry JD. Transformation of monomorphic and pleomorphic Trypanosoma brucei. Methods Mol Biol. 2004;262:5386. doi: 10.1385/1-59259-761-0:053. PubMed PMID: 14769956.

26. Dobbelaere DA, Kuenzi P. The strategies of the Theileria parasite: a new twist in host-pathogen interactions. Curr Opin Immunol. 2004;16(4):524-30. doi: 10.1016/j.coi.2004.05.009. PubMed PMID: 15245750.

27. Dobbelaere DA, Coquerelle TM, Roditi IJ, Eichhorn M, Williams RO. Theileria parva infection induces autocrine growth of bovine lymphocytes. Proc Natl Acad Sci U S A. 1988;85(13):4730-4. PubMed PMID: 3133661; PubMed Central PMCID: PMCPMC280509.

28. Dobbelaere DA, Roditi IJ, Coquerelle TM, Kelke C, Eichhorn M, Williams RO. Lymphocytes infected with Theileria parva require both cell-cell contact and growth factor to proliferate. Eur J Immunol. 1991;21(1):89-95. doi: 10.1002/eji.1830210114. PubMed PMID: 1899385.

29. Twu O, de Miguel N, Lustig G, Stevens GC, Vashisht AA, Wohlschlegel JA, et al. Trichomonas vaginalis exosomes deliver cargo to host cells and mediate hostratioparasite interactions. PLoS Pathog. 2013;9(7):e1003482. doi: 10.1371/journal.ppat.1003482. PubMed PMID: 23853596; PubMed Central PMCID: PMCPMC3708881.

30. Couper KN, Barnes T, Hafalla JC, Combes V, Ryffel B, Secher T, et al. Parasitederived plasma microparticles contribute significantly to malaria infection-induced inflammation through potent macrophage stimulation. PLoS Pathog. 2010;6(1):e1000744. doi: 10.1371/journal.ppat.1000744. PubMed PMID: 20126448; PubMed Central PMCID: PMCPMC2813278.

31. Silverman JM, Clos J, Horakova E, Wang AY, Wiesgigl M, Kelly I, et al. Leishmania exosomes modulate innate and adaptive immune responses through effects on monocytes and dendritic cells. J Immunol. 2010;185(9):5011-22. doi: 10.4049/jimmunol.1000541. PubMed PMID: 20881185.

32. Silverman JM, Clos J, de'Oliveira CC, Shirvani O, Fang Y, Wang C, et al. An exosome-based secretion pathway is responsible for protein export from Leishmania 
and communication with macrophages. J Cell Sci. 2010;123(Pt 6):842-52. doi: 10.1242/jcs.056465. PubMed PMID: 20159964.

33. Lambertz U, Oviedo Ovando ME, Vasconcelos EJ, Unrau PJ, Myler PJ, Reiner NE. Small RNAs derived from tRNAs and rRNAs are highly enriched in exosomes from both old and new world Leishmania providing evidence for conserved exosomal RNA Packaging. BMC Genomics. 2015;16:151. doi: 10.1186/s12864-015-1260-7. PubMed PMID: 25764986; PubMed Central PMCID: PMCPMC4352550.

34. Atayde VD, Aslan H, Townsend S, Hassani K, Kamhawi S, Olivier M. Exosome Secretion by the Parasitic Protozoan Leishmania within the Sand Fly Midgut. Cell Rep. 2015;13(5):957-67. doi: 10.1016/j.celrep.2015.09.058. PubMed PMID: 26565909; PubMed Central PMCID: PMCPMC4644496.

35. Fichorova RN, Trifonova RT, Gilbert RO, Costello CE, Hayes GR, Lucas JJ, et al. Trichomonas vaginalis lipophosphoglycan triggers a selective upregulation of cytokines by human female reproductive tract epithelial cells. Infect Immun. 2006;74(10):5773-9. doi: 10.1128/IAI.00631-06. PubMed PMID: 16988255; PubMed Central PMCID: PMCPMC1594934.

36. de Miguel N, Lustig G, Twu O, Chattopadhyay A, Wohlschlegel JA, Johnson PJ. Proteome analysis of the surface of Trichomonas vaginalis reveals novel proteins and strain-dependent differential expression. Mol Cell Proteomics. 2010;9(7):1554-66. doi: 10.1074/mcp.M000022-MCP201. PubMed PMID: 20467041; PubMed Central PMCID: PMCPMC2938091.

37. Neves RF, Fernandes AC, Meyer-Fernandes JR, Souto-Padron T. Trypanosoma cruzi-secreted vesicles have acid and alkaline phosphatase activities capable of increasing parasite adhesion and infection. Parasitol Res. 2014;113(8):2961-72. doi: 10.1007/s00436-014-3958-x. PubMed PMID: 24906990.

38. Bayer-Santos E, Aguilar-Bonavides C, Rodrigues SP, Cordero EM, Marques AF, Varela-Ramirez A, et al. Proteomic analysis of Trypanosoma cruzi secretome: characterization of two populations of extracellular vesicles and soluble proteins. J Proteome Res. 2013;12(2):883-97. doi: 10.1021/pr300947g. PubMed PMID: 23214914. 39. Bayer-Santos E, Lima FM, Ruiz JC, Almeida IC, da Silveira JF. Characterization of the small RNA content of Trypanosoma cruzi extracellular vesicles. Mol Biochem Parasitol. 2014;193(2):71-4. doi: 10.1016/j.molbiopara.2014.02.004. PubMed PMID: 24583081; PubMed Central PMCID: PMCPMC4058860.

40. Trocoli Torrecilhas AC, Tonelli RR, Pavanelli WR, da Silva JS, Schumacher RI, de Souza W, et al. Trypanosoma cruzi: parasite shed vesicles increase heart parasitism and generate an intense inflammatory response. Microbes Infect. 2009;11(1):29-39. doi: 10.1016/j.micinf.2008.10.003. PubMed PMID: 19028594.

41. Garcia-Silva MR, Cabrera-Cabrera F, das Neves RF, Souto-Padron T, de Souza W, Cayota A. Gene expression changes induced by Trypanosoma cruzi shed microvesicles in mammalian host cells: relevance of tRNA-derived halves. Biomed Res Int.

2014;2014:305239. doi: 10.1155/2014/305239. PubMed PMID: 24812611; PubMed Central PMCID: PMCPMC4000953.

42. Garcia-Silva MR, das Neves RF, Cabrera-Cabrera F, Sanguinetti J, Medeiros LC, Robello C, et al. Extracellular vesicles shed by Trypanosoma cruzi are linked to small RNA 
pathways, life cycle regulation, and susceptibility to infection of mammalian cells. Parasitol Res. 2014;113(1):285-304. doi: 10.1007/s00436-013-3655-1. PubMed PMID: 24241124.

43. Stierhof YD, Bates PA, Jacobson RL, Rogers ME, Schlein Y, Handman E, et al. Filamentous proteophosphoglycan secreted by Leishmania promastigotes forms gel-like three-dimensional networks that obstruct the digestive tract of infected sandfly vectors. Eur J Cell Biol. 1999;78(10):675-89. doi: 10.1016/S0171-9335(99)80036-3. PubMed PMID: 10569240.

44. Bates PA. Transmission of Leishmania metacyclic promastigotes by phlebotomine sand flies. Int J Parasitol. 2007;37(10):1097-106. doi: 10.1016/j.ijpara.2007.04.003. PubMed PMID: 17517415; PubMed Central PMCID: PMCPMC2675784.

45. Peters NC, Egen JG, Secundino N, Debrabant A, Kimblin N, Kamhawi S, et al. In vivo imaging reveals an essential role for neutrophils in leishmaniasis transmitted by sand flies. Science. 2008;321(5891):970-4. doi: 10.1126/science.1159194. PubMed PMID: 18703742; PubMed Central PMCID: PMCPMC2606057.

46. Belkaid Y, Kamhawi S, Modi G, Valenzuela J, Noben-Trauth N, Rowton E, et al. Development of a natural model of cutaneous leishmaniasis: powerful effects of vector saliva and saliva preexposure on the long-term outcome of Leishmania major infection in the mouse ear dermis. J Exp Med. 1998;188(10):1941-53. PubMed PMID: 9815271; PubMed Central PMCID: PMCPMC2212417.

47. Rogers ME, Bates PA. Leishmania manipulation of sand fly feeding behavior results in enhanced transmission. PLoS Pathog. 2007;3(6):e91. doi:

10.1371/journal.ppat.0030091. PubMed PMID: 17604451; PubMed Central PMCID: PMCPMC1904410.

48. Rogers ME, IIg T, Nikolaev AV, Ferguson MA, Bates PA. Transmission of cutaneous leishmaniasis by sand flies is enhanced by regurgitation of fPPG. Nature. 2004;430(6998):463-7. doi: 10.1038/nature02675. PubMed PMID: 15269771; PubMed Central PMCID: PMCPMC2835460.

49. Rogers $M$, Kropf $P$, Choi BS, Dillon R, Podinovskaia M, Bates $P$, et al.

Proteophosophoglycans regurgitated by Leishmania-infected sand flies target the Larginine metabolism of host macrophages to promote parasite survival. PLoS Pathog. 2009;5(8):e1000555. doi: 10.1371/journal.ppat.1000555. PubMed PMID: 19696894; PubMed Central PMCID: PMCPMC2722086.

50. Ghosh J, Bose M, Roy S, Bhattacharyya SN. Leishmania donovani targets Dicer1 to downregulate miR-122, lower serum cholesterol, and facilitate murine liver infection. Cell Host Microbe. 2013;13(3):277-88. doi: 10.1016/j.chom.2013.02.005. PubMed PMID: 23498953; PubMed Central PMCID: PMCPMC3605572.

51. Geiger A, Hirtz C, Becue T, Bellard E, Centeno D, Gargani D, et al. Exocytosis and protein secretion in Trypanosoma. BMC Microbiol. 2010;10:20. doi: 10.1186/1471-218010-20. PubMed PMID: 20102621; PubMed Central PMCID: PMCPMC3224696.

52. Szempruch AJ, Sykes SE, Kieft R, Dennison L, Becker AC, Gartrell A, et al. Extracellular Vesicles from Trypanosoma brucei Mediate Virulence Factor Transfer and 
Cause Host Anemia. Cell. 2016;164(1-2):246-57. doi: 10.1016/j.cell.2015.11.051.

PubMed PMID: 26771494; PubMed Central PMCID: PMCPMC4715261.

53. Murray M, Dexter TM. Anaemia in bovine African trypanosomiasis. A review. Acta Trop. 1988;45(4):389-432. PubMed PMID: 2907267.

54. Naessens J. Bovine trypanotolerance: A natural ability to prevent severe anaemia and haemophagocytic syndrome? Int J Parasitol. 2006;36(5):521-8. doi:

10.1016/j.ijpara.2006.02.012. PubMed PMID: 16678182.

55. Naessens J, Leak SG, Kennedy DJ, Kemp SJ, Teale AJ. Responses of bovine chimaeras combining trypanosomosis resistant and susceptible genotypes to experimental infection with Trypanosoma congolense. Vet Parasitol. 2003;111(2-3):12542. PubMed PMID: 12531289.

56. Rupp I, Sologub L, Williamson KC, Scheuermayer M, Reininger L, Doerig C, et al. Malaria parasites form filamentous cell-to-cell connections during reproduction in the mosquito midgut. Cell Res. 2011;21(4):683-96. doi: 10.1038/cr.2010.176. PubMed PMID: 21173797; PubMed Central PMCID: PMCPMC3072464.

57. Imhof S, Fragoso C, Hemphill A, von Schubert C, Li D, Legant W, et al. Flagellar fusion and protein exchange in trypanosomes; a new form of cell-cell communication. F1000Research. 2016;in press.

58. Peacock L, Ferris V, Sharma R, Sunter J, Bailey M, Carrington M, et al. Identification of the meiotic life cycle stage of Trypanosoma brucei in the tsetse fly. Proc Natl Acad Sci U S A. 2011;108(9):3671-6. doi: 10.1073/pnas.1019423108. PubMed PMID: $21321215 ;$ PubMed Central PMCID: PMCPMC3048101.

59. Sadlova J, Yeo M, Seblova V, Lewis MD, Mauricio I, Volf $P$, et al. Visualisation of Leishmania donovani fluorescent hybrids during early stage development in the sand fly vector. PLoS One. 2011;6(5):e19851. doi: 10.1371/journal.pone.0019851. PubMed PMID: 21637755; PubMed Central PMCID: PMCPMC3103508.

60. Calvo-Alvarez E, Alvarez-Velilla R, Jimenez M, Molina R, Perez-Pertejo Y, BalanaFouce $R$, et al. First evidence of intraclonal genetic exchange in trypanosomatids using two Leishmania infantum fluorescent transgenic clones. PLoS Negl Trop Dis. 2014;8(9):e3075. doi: 10.1371/journal.pntd.0003075. PubMed PMID: 25188587; PubMed Central PMCID: PMCPMC4154677.

61. Gluenz E, Ginger ML, McKean PG. Flagellum assembly and function during the Leishmania life cycle. Curr Opin Microbiol. 2010;13(4):473-9. doi: 10.1016/j.mib.2010.05.008. PubMed PMID: 20541962.

62. Bassler BL, Losick R. Bacterially speaking. Cell. 2006;125(2):237-46. doi: 10.1016/j.cell.2006.04.001. PubMed PMID: 16630813.

63. Benomar S, Ranava D, Cardenas ML, Trably E, Rafrafi Y, Ducret A, et al. Nutritional stress induces exchange of cell material and energetic coupling between bacterial species. Nat Commun. 2015;6:6283. doi: 10.1038/ncomms7283. PubMed PMID: 25704114.

64. Pande S, Shitut S, Freund L, Westermann M, Bertels F, Colesie C, et al. Metabolic cross-feeding via intercellular nanotubes among bacteria. Nat Commun. 2015;6:6238. doi: 10.1038/ncomms7238. PubMed PMID: 25703793. 
65. Prindle A, Liu J, Asally M, Ly S, Garcia-Ojalvo J, Suel GM. Ion channels enable electrical communication in bacterial communities. Nature. 2015;527(7576):59-63. doi: 10.1038/nature15709. PubMed PMID: 26503040.

\section{FIGURE LEGEND}

Different types of signals produced by parasites and their effects. (1) Trypanosoma brucei; (2) Trichomonas vaginalis; (3) Plasmodium species; (4) Leishmania species; (5) Trypanosoma cruzi; (6) Theileria parva. The nature of the migration and repellents causing social motility of $T$. brucei are not known. 


\section{EFFECT ON PARASITE}

differentiation (1)

adhesion (2)

differentiation (3)

increased parasite titre (4) change of host cell type (3)

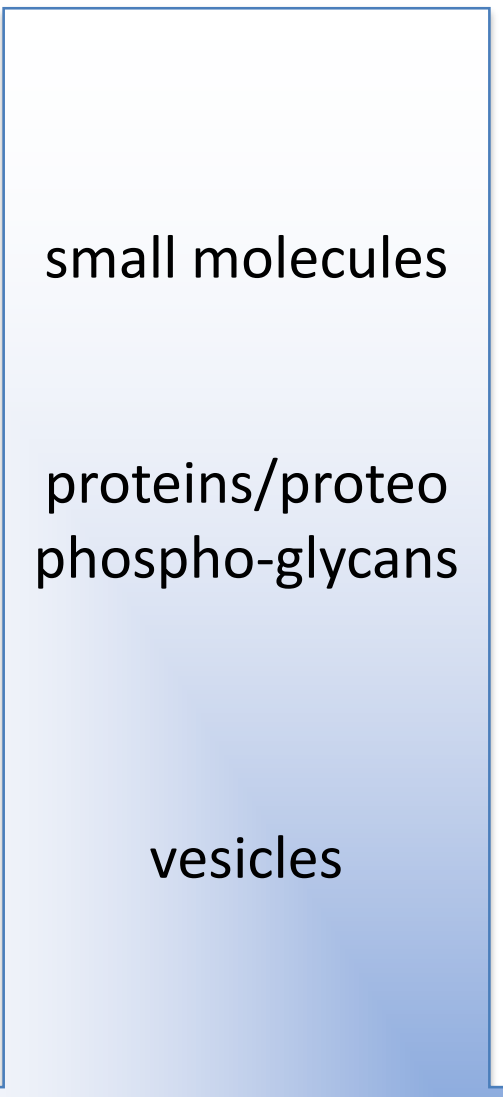

\section{EFFECT ON HOST}

recruitment of host cells (4) metabolic changes (4)

altered cytokine response $(2,3,4,5)$ modulation of transcriptome $(4,5)$ erythrocyte lysis (1) modulation of serum components (4) adhesion (3)

proliferation (6) protein exchange (1) 